\title{
Effectiveness of Lifestyle Health Promotion Interventions for Nurses: Protocol for a Systematic Review
}

\author{
Holly Blake, $P h D^{1^{*}}$, Melanie Narayanasamy, PhD², Mark E Batt, MB DM FFSEM ${ }^{3}$ and Kamlesh Khunti \\ $\mathrm{MD}, \mathrm{PhD}, \mathrm{FMedSCi}{ }^{4}$
}

\begin{abstract}
${ }^{1}$ Associate Professor of Behavioural Science, School of Health Sciences, Queen's Medical Centre, Nottingham, England, UK
\end{abstract}

${ }^{2}$ Research Fellow, School of Health Sciences, Queen's Medical Centre, Nottingham, England, UK

${ }^{3}$ Consultant in Sport and Exercise Medicine, Centre for Sports Medicine, Nottingham University Hospitals NHS Trust, Nottingham, England, UK

${ }^{4}$ Professor of Primary Care Diabetes and Vascular Medicine, Diabetes Research Centre, University of Leicester, Leicester, England, UK

\section{Background}

\section{Behavioural health risk factors of working age nurses}

Risky lifestyle behaviours amongst nurses have been well documented. Studies have shown non-adherence among nurses to national public health guidelines for physical activity, diet, smoking, and alcohol consumption [1-10]. Obesity and overweight are prevalent in nurses. A study of Scottish nurses showed that $69 \%$ were overweight or obese, which was higher than rates for other healthcare professionals, unqualified care staff and those in non-health related occupations [11]. Recent analysis of data from the national Health Survey for England revealed that $25 \%$ of English nurses are obese (body mass index $\geq 30.0$ ), again, with rates higher than those for other healthcare professionals [12]. Unhealthy lifestyle behaviours commonly cluster together, increasing nurse's health risks; for example, physical inactivity in nurses has been associated with other behavioural risk factors such as smoking and obesity [2]. Mental health issues are common within the nursing profession including high rates of work-related stress, burnout, anxiety and depression [13-16].

While nurses through their training are educated on the importance of physical and mental health and its relationship with morbidity and mortality, they do not necessarily transfer this knowledge to their own lifestyle practices $[4,5,17,18]$. Reasons for engagement in unhealthy lifestyle choices among the nursing workforce are complex. They may be related to individual factors such as personal motivation [18], jobrelated factors such as working long hours, heavy workloads and shift work $[14,17]$ or environmental factors such as hospital catering availability [14].

\section{Implications of unhealthy lifestyle behaviours}

Unhealthy lifestyle choices increase the risk of chronic dis- ease and sickness absenteeism. In addition to the individual burden of physical and mental ill-health, staff sickness absenteeism incurs significant financial burden to the National Health Service (NHS). Approximately $£ 1$ in every $£ 40$ of the NHS budget is spent on NHS staff absence, which is estimated at $£ 2.4$ bn per year before agency costs are taken into account [19]. In addition, presenteeism- working in poor health - is an escalating issue in the NHS $[20,21]$, and costs twice as much as absenteeism [22].

Health and wellbeing of nurses may impact on productivity and the quality of patient care [23]. Absenteeism and high turnover reduce continuity of care for patients [24]. Presenteeism contributes to unsafe care [25]. Furthermore, nurses report that their own health behaviours may influence their attitude to health promoting [26], and their willingness and perceived ability to promote healthy lifestyles to patients [2729]. A systematic review and meta-analysis found that nurses who smoked were $13 \%$ less likely to encourage patients to quit smoking and $25 \%$ less likely to organise smoking cessation follow-up [29]. Nurses who are overweight have reported a negative impact of their weight on their work performance [4]. This suggests that nurses own lifestyle choices may impact on care quality, and ultimately, patient clinical outcomes.

*Corresponding author: Holly Blake, BA (Hons), PhD CPsychol SFHEA, Associate Professor of Behavioural Science, School of Health Sciences, Queen's Medical Centre, Nottingham, NG7 2HA, UK, Tel: +44-(0)-115-82-31049

Accepted: April 12, 2019

Published online: May 25, 2019

Citation: Blake H, Narayanasamy M, Batt ME, et al. (2019) Effectiveness of Lifestyle Health Promotion Interventions for Nurses: Protocol for a Systematic Review. J Nurs Pract 3(1):81-85 
Citation: Blake H, Narayanasamy M, Batt ME, et al. (2019) Effectiveness of Lifestyle Health Promotion Interventions for Nurses: Protocol for a Systematic Review. J Nurs Pract 3(1):81-85

The UK government has called for immediate action to improve the health of the NHS workforce, in particular frontline care staff $[30,31]$. However, nurses can be hard to reach, often due to work-related barriers to accessing lifestyle intervention, such as shift work [32]. There needs to be a better understanding of the types of lifestyle interventions that appeal to nurses, and which interventions can impact most positively on individual outcomes (i.e. lifestyle behaviour; physical and psychological health), and organisational outcomes (i.e. employee engagement, job satisfaction, performance, productivity, sickness absence, patient safety and care).

\section{Previous systematic reviews}

This systematic review builds on previous reviews in this field. Chan and Perry [6] published a similar systematic review with studies included up to 2011, although new evidence has emerged following increased government and media attention around the impact of nurses' health on compassion and care quality. Psychological outcomes of lifestyle interventions (i.e. stress) were not previously considered, yet there is a known relationship between lifestyle behaviours and psychological wellbeing. For example, Jordan, et al. identified that nurses who had higher stress levels were more likely to engage in unhealthy eating as a coping strategy. The prevalence of mental health issues within the healthcare workforce is high [14,33,34]. A study by Sarafis, et al. [35] found that occupational stress impacted on the quality of life of nurses, influenced patient outcomes, and could be considered to be a predictor of nurses' caring behaviour implementation. Chan and Perry's [6] review did not explore organisational outcomes of lifestyle intervention and therefore it is not clear how lifestyle interventions for nurses may impact on outcomes such as work engagement, job satisfaction and sickness absenteeism. However, researchers have documented links between unhealthy lifestyle behaviours, stress, work engagement and job satisfaction $[3,16,36,37]$. There are complex organisational issues that impact on nurses' stress, NHS costs and care quality, including staffing shortages and workload, turnover, failure to retain staff, and shift patterns. However, it has been suggested that healthier working environments offering lifestyle intervention for nurses may generate a workforce with lower stress levels, greater employee satisfaction, greater retention of the nursing workforce, and ultimately may improve the quality of care being provided [3].

Systematic reviews around health promotion interventions addressing both individual (including physical and psychological health outcomes) and organisational outcomes of working age nurses have not yet been conducted. Prior systematic reviews have focused on one specific outcome and/ or have restricted the focus to one specific type of nursing job role [38-40]. There is a clear need for a systematic review of lifestyle interventions targeting the nursing workforce more broadly, to determine which types of interventions impact most positively on individual outcomes (i.e. health behaviours; physical and psychological health), and organisational outcomes of interest to healthcare employers (i.e. employee engagement, job satisfaction, sickness absence).

\section{Aims}

The primary aim of this systematic review is to establish the efficacy of lifestyle health promotion interventions intended to improve behavioural health risk factors and/or behavioural or clinical outcomes of working-age nurses. The secondary aim is to identify the efficacy of these interventions in improving organisational outcomes.

\section{Methods}

This is a systematic review undertaken in line with guidance for reviews in health care [41]. The review was registered in PROSPERO on 29 ${ }^{\text {th }}$ May 2018 (CRD42018098642).

\section{Eligibility criteria}

\section{[i] Types of studies}

Due to the paucity of evidence identified in a previous review, controlled trials, non-controlled intervention studies and reviews of intervention studies will be eligible for inclusion. Non-controlled intervention studies may include interrupted time series studies, cohort studies, cross-sectional studies and case series.

\section{[ii] Types of participants}

Studies will include working-age nurses defined as adults in employment holding a recorded nursing qualification. In studies with mixed participant groups, nurses must constitute at least $50 \%$ of the target population. Studies primarily targeting student nurses or retired nurses are excluded.

\section{[iii] Context/Setting}

Any healthcare workplace setting in which nurses with a recorded nursing qualification are accessed. Studies from any country will be included.

\section{[iv] Types of interventions}

Behavioural and/or educational interventions, either alone or in combination, will be included, which are aimed at improving any of (but not limited to):

- Health risk factors: overweight or obesity, diet, physical activity, smoking habits, problem drinking.

- Clinical health outcomes: type 2 diabetes, stroke, chronic heart disease, cancers, hypertension.

- Psychological health outcomes: stress, anxiety, depression, burnout, self-efficacy.

- Organisational outcomes: job satisfaction, organisational commitment, employee engagement, sickness absenteeism, early retirement, performance, productivity, staff retention, staff turnover, patient safety and care.

\section{[v] Comparator(s)/control}

Since the purpose of the review is to identify lifestyle health promotion interventions (as well as to assess efficacy of these) it is not possible to define comparator(s)/control in advance. However, for any controlled trial studies identified, we will describe what interventions the control group received. If no alternative interventions were used for the 
Citation: Blake H, Narayanasamy M, Batt ME, et al. (2019) Effectiveness of Lifestyle Health Promotion Interventions for Nurses: Protocol for a Systematic Review. J Nurs Pract 3(1):81-85

control group, this will also be stated. For any non-controlled studies identified, comparator(s)/control is not applicable.

\section{[vi] Types of outcome measures}

Studies will assess outcomes either as changes in health knowledge, health behaviours, disease risk factor indices, related mortality and morbidity or changes in organisational outcomes (including job-related factors, patient safety and care). Eligible risk factor changes and clinical outcomes will be specified. This may include scores from baseline to last available follow-up. Outcome measures may include (though not limited to):

- Changes to weight, BMI, waist or other anthropometric indices

- Changes to diet (e.g. intake of fruit and vegetables; lipid and cholesterol levels)

- Changes in levels of physical activity (e.g. frequency, duration, intensity)

- Changes to smoking habits (e.g. number of cigarettes smoked per day, cessation attempts)

- Changes in alcohol consumption

- Changes to psychological health (e.g. levels of stress, anxiety, burnout, depression, self-efficacy)

- Clinical outcomes (e.g. related morbidity; hypertension with changes in systolic and/or diastolic values; type 2 diabetes with changes in incidence prevalence or indices of glycaemic control such as HbA1c values. Longer-term related morbidity or mortality included incidence of Acute Coronary Syndrome; renal or liver failure; peripheral vascular disease; cerebrovascular disease; incidence of neurovascular complications of type 2 diabetes; cancers)

Table 1: Example search strategy.

\begin{tabular}{|l|l|}
\hline & Search Term \\
\hline 1. & Physical Activity/ \\
\hline 2. & Exercise/ \\
\hline 3. & (physical activity or exercise). af \\
\hline 4. & Diet/ \\
\hline 5. & Obesity/ \\
\hline 6. & (diet or obesity or weight).af \\
\hline 7. & Smoking/ \\
\hline 8. & Smoking Cessation/ \\
\hline 9. & (smok ${ }^{*}$ or cigarette\$ or nicotine or tobacco).af \\
\hline 10. & Alcohol Drinking/ \\
\hline 11. & alcohol drinking or alcohol consum ${ }^{*}$ af \\
\hline 12. & 1 or 2 or 3 or 4 or 5 or 6 or 7 or 8 or 9 or 10 or 11 \\
\hline 13. & Hypertension/ \\
\hline 14. & Diabetes Mellitus, Type $2 /$ \\
\hline 15. & Acute Coronary Syndrome/ \\
\hline
\end{tabular}

- Organisational outcomes (e.g. changes in job satisfaction, organisational commitment, employee engagement, sickness absenteeism, early retirement rates or intentions, performance measures, productivity, staff retention rates, staff turnover rates, measures of patient safety and care).

\section{Search strategy}

Seven electronic databases will be searched (using MeSH and free text search terms) for eligible studies including the Cochrane Central Register of Controlled Trials; MEDLINE and PubMed; EMBASE; CINAHL; PsycINFO; and BioMed Central from January 2000 to August 2017. Reference lists of identified records and relevant reviews will be checked. Only studies published in English will be included. A proposed search strategy is included in Table 1.

\section{Selection processes}

Two reviewers will independently perform study selection. A reference manager program will be used to store references and this will be used to identify any duplicated records. Once duplications have been removed, the titles and abstracts of remaining records will be screened. Full texts will be sought for records which clearly refer to behavioural and/ or educational lifestyle interventions for working age nurses. These full texts will then be assessed for eligibility, taking into account intervention type, study population, outcomes reported, and language. Agreement will be reached through discussion, and if consensus cannot be reached, a third reviewer will make the final decision.

\section{Data extraction}

Data extraction will be performed independently by two reviewers and agreement will be reached through discussion. If consensus cannot be reached, a third reviewer will

\begin{tabular}{|c|c|}
\hline 16. & Acute Kidney Injury/ \\
\hline 17. & Liver Failure/ \\
\hline 18. & Liver Failure, Acute/ \\
\hline 19. & Peripheral Vascular Diseases/ \\
\hline 20. & Cerebrovascular Disorders/ \\
\hline 21. & $\begin{array}{l}\text { (hypertension or diabetes or coronary or renal } \\
\text { failure or kidney failure or liver failure or cancer). } \\
\text { af }\end{array}$ \\
\hline 22. & $\begin{array}{l}13 \text { or } 14 \text { or or } 15 \text { or } 16 \text { or } 17 \text { or } 18 \text { or } 19 \text { or } 20 \text { or } \\
21\end{array}$ \\
\hline 23. & Mental Health/ \\
\hline 24. & Stress Disorders, Traumatic, Acute/ \\
\hline 25. & Anxiety/ \\
\hline 26. & Burnout, Professional/ \\
\hline 27. & Compassion Fatigue/ \\
\hline 28. & Depression/ \\
\hline 29. & Depressive Disorder/ \\
\hline 30. & Self-Efficacy/ \\
\hline
\end{tabular}




\begin{tabular}{|c|c|}
\hline 31. & $\begin{array}{l}\text { mental health or mental illness or psychological } \\
\text { or psychological wellbeing or stress or anxiety or } \\
\text { burnout or depression or self-efficacy or self ADJ } \\
\text { efficacy }\end{array}$ \\
\hline 32. & 23 or 24 or 25 or 26 or 27 or 28 or 29 or 31 \\
\hline 33. & Absenteeism/ \\
\hline 34. & Job Satisfaction/ \\
\hline 35. & Work Engagement/ \\
\hline 36. & Work Performance/ \\
\hline 37. & Patient Safety/ \\
\hline 38. & $\begin{array}{l}\text { sicknessabsen* or absen* or job satisfaction or } \\
\text { employee engagement or work performance or } \\
\text { staff retention or staff turnover or patient safety } \\
\text { or quality of care or patient care.af }\end{array}$ \\
\hline 39. & 33 or 34 or 35 or 36 or 37 or 38 \\
\hline 40. & $\begin{array}{l}\text { (intervention or lifestyle or behavior }{ }^{*} \text { or behavior } \\
\text { change intervention* or behavior* change } \\
\text { technique*).af. }\end{array}$ \\
\hline 41. & $\begin{array}{l}\text { (nurs\$ not in-patient\$ not inpatient\$ not } \\
\text { patient\$).af. }\end{array}$ \\
\hline 42. & 12 or 22 or 32 or 39 \\
\hline 43. & 40 and 41 and 42 \\
\hline 44. & $\begin{array}{l}\text { limit } 43 \text { to (human and English language) and yr = } \\
\text { "2000-Current" }\end{array}$ \\
\hline
\end{tabular}

make the final decision. Participant, setting, intervention, and outcome measurement details will be extracted from each study. Methodological features of trials and reviews will be described using the CONSORT and PRISMA checklists [42] respectively.

\section{Quality appraisal}

Two reviewers will independently review and critique retrieved papers. Risk of bias will be assessed using the Cochrane Handbook classification [43]. Risk of selection, performance, detection, attrition and reporting bias will be assessed. Agreement will be reached through discussion, and if consensus cannot be reached, a third reviewer will make the final decision.

\section{Methods of synthesis}

Studies will be summarised narratively, which is acknowledged as an appropriate approach to take when assessing data from different study designs [44]. We will provide a narrative synthesis of the findings from the included studies, structured around the type of intervention, target population characteristics, type of outcome and intervention content. We will provide summaries of intervention effects for each study. If included studies are sufficiently homogenous, a statistical meta-analytic summary will be produced.

\section{Analysis of Subgroups or Subsets}

It is not possible to specify sub-groups at the outset based, for example, on factors such as age or qualifications.
There may be some intervention studies that target specific populations (such as older nurses, newly qualified nurses, or nurses working in particular settings such as acute or community care). However, we intend that the review itself will identify the range of interventions offered and we will be able to describe the target nursing populations and their settings, the outcomes assessed and evidence of effectiveness, narratively. Narrative synthesis will also enable us to explore different types of study designs.

\section{Conclusion}

The published evidence-base for lifestyle interventions specifically targeting (or including) nurses is currently limited. This systematic review will be the first to assess the effectiveness of lifestyle health promotion interventions with regards both individual (such as physical and psychological health) and organisational outcomes (such as job satisfaction and absenteeism). The review will provide insight into the range of interventions being offered for nurses and how effective they are at improving health, health behaviours, and psychological and/or clinical/health outcomes in nurses. This will help to inform the design and implementation of health and wellbeing services to support nurses and therefore help to improve future workplace health service provision and quality.

\section{Funding Sources/Sponsors}

This study is funded by the National Institute for Health Research (NIHR) Collaboration for Leadership in Applied Health Research and Care East Midlands (CLAHRC EM). The views expressed are those of the author(s) and not necessarily those of the NIHR or the Department of Health and Social Care.

\section{References}

1. Schneider A, Bak M, Mahoney C, et al. (2019) Health-related behaviours of nurses and other healthcare professionals: $A$ cross-sectional study using the Scottish Health Survey. J Adv Nurs 75: 1239-1251.

2. Al-Tannir MA, Kobrosly SY, Elbakri NK, et al. (2017) Prevalence and predictors of physical exercise among nurses- A crosssectional study. Saudi Med J 38: 209-212.

3. Jordan TR, Khubchandani J, Wiblishauser M (2016) The Impact of Perceived Stress and Coping Adequacy on the Health of Nurses: A Pilot Investigation. Nursing Research and Practice, Article ID 5843256.

4. Phiri LP, Draper CE, Lambert EV, et al. (2014) Nurses' lifestyle behaviours, health priorities and barriers to living a healthy lifestyle: a qualitative descriptive study. BMC Nurs 13: 38.

5. Blake H, Harrison C (2013) Health behaviours and attitudes towards being role models. British Journal of Nursing 22: 86-94.

6. Chan CW, Perry L (2012) Lifestyle health promotion interventions for the nursing workforce: a systematic review. J Clin Nurs 21: 2247-2261.

7. Blake H, Malik S, Mo PKH, et al. (2011) "Do as I say, but not as I do": Are next generation nurses role models for health? Perspectives in Public Health 131: 231-239.

8. Blake H, Mo PKH, Lee S, et al. (2012) Health in the NHS: lifestyle behaviours of hospital employees. Perspectives in Public Health 132: 213-215. 
Citation: Blake H, Narayanasamy M, Batt ME, et al. (2019) Effectiveness of Lifestyle Health Promotion Interventions for Nurses: Protocol for a Systematic Review. J Nurs Pract 3(1):81-85

9. Mo PKH, Blake H, Batt ME (2011) Getting healthcare staff more active: the mediating role of self-efficacy. British Journal of Health Psychology 16: 690-706.

10. Malik S, Blake H, Batt ME (2011) How healthy are our nurses? New and registered nurses compared. Br J Nurs 20: 489-496.

11. Kyle RG, Wills J, Mahoney C, et al. (2016) Obesity prevalence among healthcare professionals in England: a cross-sectional study using the Health Survey for England, BMJ Open 12 e018498.

12. Kyle RG, Neall RA, Atherton IM (2017) Prevalence of overweight and obesity among nurses in Scotland: A cross-sectional study using the Scottish Health Survey. Int J Nurs Stud 53: 126-133.

13. Jackson J, Vandall-Walker V, Vanderspank-Wright B, et al. (2018) Burnout and resilience in critical care nurses: A grounded theory of Managing Exposure. Intensive Crit Care Nurs 48: 28-35.

14. Haplin Y, Terry LM, Curzio J (2017) A longitudinal, mixed methods investigation of newly qualified nurses' workplace stressors and stress experiences during transition. J Adv Nurs 73: 2577-2586.

15. Adriaenssens J, De Gucht V, Maes S (2015) Determinants and prevalence of burnout in emergency nurses: A systematic review of 25 years of research. Int J Nurs Stud 52: 649-661.

16. Khamisa N, Oldenburg B, Peltzer K, et al. (2015) Work Related Stress, Burnout, Job Satisfaction and General Health of Nurses. Int J Environ Res Public Health 12: 652-666.

17. Ross A, Bevans M, Brooks AT, et al. (2017) Nurses and HealthPromoting Behaviors: Knowledge May Not Translate Into SelfCare. AORN Journal 105: 267-275.

18. Miller SK, Alpert PT, Cross CL (2008) Overweight and obesity in nurses, advanced practice nurses, and nurse educators. J Am Acad Nurse Pract 20: 259-265.

19. NHS England (2015) Simon Stevens announces major drive to improve health in NHS workplace.

20. West MA, Dawson JF (2012) Employee engagement and NHS performance. The King's Fund.

21. Personnel Today (2009) NHS sickness absence: HR must first address culture of "presenteeism".

22. Personnel Today (2015) "Presenteeism costs twice as much as sickness absence".

23. Letvak SA, Ruhm C, Lane S (2011) The Impact of Nurses' Health on Productivity and Quality of Care. J Nurs Adm 41: 162-167.

24. Davey MM, Cummings G, Newburn-Cook CV, et al. (2009) Predictors of nurse absenteeism in hospitals: a systematic review. J Nurs Manag 17: 312-330.

25. Letvak SA, Ruhm CJ, Gupta SN (2012) Nurses' presenteeism and its effects on self-reported quality of care and costs. Am J Nurs 112: 30-38.

26. Blake H, Stanulewicz N, Griffiths K (2016) Healthy lifestyle behaviours predict health promotion attitudes in pre-registered nurses. J Nurs Educ 56: 94-103.

27. Priano SM, Hong OS, Chen J-L (2018) Lifesyles and Health-
Related Outcomes of US Hospital Nurses: A Systematic Review. Nurs Outlook 66: 66-67.

28. Blake H, Patterson J (2015) Paediatric nurses' attitudes towards the promotion of healthy eating in children and their families. $\mathrm{Br}$ J Nurs 24: 108-112.

29. Duaso MJ, Bakhshi S, Mujika A, et al. (2017) Nurses' smoking habits and their professional smoking cessation practices. A systematic review and meta-analysis. Int J Nurs Stud 67: 3-11.

30. NHS England (2016) NHS staff health \& wellbeing: CQUIN Supplementary guidance. NHS England.

31. Prime Minister's Commission (2010) Front line care: Report by the Prime Minister's Commission on the Future of Nursing and Midwifery in England.

32. Blake H, Stanulewicz N, McGill F (2017) Predictors of physical activity and barriers to exercise in nursing and medical students. J Adv Nurs 73: 917-929.

33. Lenthall S, Wakerman J, Dollard MF, et al. (2018) Reducing occupational stress among registered nurses in very remote Australia: A participatory action research approach. Collegian 25: 181-191.

34. Stephenson J (2018) NHS staff survey shows rise in stress levels, staffing concerns and attacks. Nursing Times.

35. Sarafis P, Rousaki E, Tsounis A, et al. (2016) The impact of occupational stress on nurses' caring behaviors and their health related quality of life. BMC Nursing 15: 56.

36. Pérez-Fuentes MDC, MoleroJurado MDM, Barragán Martín AB, et al. (2018) The Mediating Role of Perceived Stress in the Relationship of Self-Efficacy and Work Engagement in Nurses. J Clin Med 8: 10.

37. Jaradat $Y M$, Nielsen MB, Kristensen $P$, et al. (2017) Shift work, mental distress and job satisfaction among Palestinian nurses. Occup Med 67: 71-74.

38. Laitila AH, Romppanen J (2017) Outcomes of interventions for nurse leaders' well-being atwork: A quantitative systematic review. J Adv Nurs 74: 34-44.

39. Halter M, Pelone F, Boiko O, et al. (2017) Interventions to Reduce Adult Nursing Turnover: A Systematic Review of Systematic Reviews. Open Nurs J 11: 108-123.

40. Duhoux A, Menear M, Charron M, et al. (2017) Interventions to promote or improve the mental health of primary care nurses: a systematic review. J Nurs Manag 25: 597-607.

41. Centre for Reviews and Dissemination (2009) Systematic Reviews: CRD's guidance for undertaking reviews in health care. York: University of York.

42. Moher D, Liberati A, Tetzlaff J, et al. (2009) Preferred Reporting Items for Systematic Reviews and Meta-Analyses: The PRISMA Statement. PLoS Med 6: e1000097.

43. Cochrane Handbook for Systematic Reviews of Interventions.

44. Ryan R (2013) Cochrane Consumers and Communication Review Group. 'Cochrane Consumersand Communication Review Group: data synthesis and analysis'. 